\title{
Numerical simulations on the motion of atoms travelling through a standing-wave light field
}

\author{
S.J.H. Petra $⿴$ K.A.H. van Leeuwen, L. Feenstra ${ }^{\dagger}$ W. Hogervorst, and W. Vassen \\ Atomic and Laser Physics Group, Laser Centre Vrije Universiteit, \\ De Boelelaan 1081, $1081 \mathrm{HV}$ Amsterdam, The Netherlands
}

\begin{abstract}
The motion of metastable helium atoms travelling through a standing light wave is investigated with a semi-classical numerical model. The results of a calculation including the velocity dependence of the dipole force are compared with those of the commonly used approach, which assumes a conservative dipole force. The comparison is made for two atom guiding regimes that can be used for the production of nanostructure arrays; a low power regime, where the atoms are focused in a standing wave by the dipole force, and a higher power regime, in which the atoms channel along the potential minima of the light field. In the low power regime the differences between the two models are negligible and both models show that, for lithography purposes, pattern widths of $150 \mathrm{~nm}$ can be achieved. In the high power channelling regime the conservative force model, predicting $100 \mathrm{~nm}$ features, is shown to break down. The model that incorporates velocity dependence, resulting in a structure size of $40 \mathrm{~nm}$, remains valid, as demonstrated by a comparison with quantum Monte-Carlo wavefunction calculations.
\end{abstract}

PACS numbers: 02.60.Cb, 32.80.Lg, 81.16.Rf

\section{INTRODUCTION}

The dipole force in a standing-wave light field has been used to create nanoscale patterns with beams of neutral atoms for many years. The standing wave can act as an array of optical lenses to focus the atoms during deposition onto a substrate, thereby creating a onedimensional structure. The first experiments using this technique were performed ten years ago with sodium 1] and chromium [2] atoms. Since then, atom lithography with aluminium [3], cesium [4], metastable argon [5], and metastable neon [6] has also been reported. For an overview of atom lithography, see Ref. 7]. The results of a number of these experiments have been compared with semi-classical numerical calculations 8, 9]. These calculations are based on a dipole force that is derived from an optical potential to focus the atoms in the standing light wave. This force is conservative and does not take into account the velocity dependence. Quantum-mechanical calculations have been performed as well 10]. These calculations do not suffer from this restriction. However, they are computationally intensive and describe the atomic motion in one dimension only.

The purpose of this paper is to investigate in detail the $3 \mathrm{D}$ atomic motion through a standing-wave light field using a semi-classical approach with a non-conservative, velocity dependent dipole force. The results of these simulations are compared with calculations using the conventional model with a conservative dipole force. The model presented in this paper follows the approach of Minogin and Serimaa 11], where the dipole force is rep-

\footnotetext{
*Electronic address: stefan@nat.vu.nl

${ }^{\dagger}$ Present address: Physikalisches Institut, Universität Heidelberg, Philosophenweg 12, 69120 Heidelberg, Germany.
}

resented in the form of a Fourier series, with coefficients that are calculated with a continued fraction method. Previously, a similar model was used to calculate a onedimensional beam profile of sodium atoms in the far field 12, 13. The model that is presented here describes a full $3 \mathrm{D}$ simulation of the motion of metastable helium atoms through a standing light wave for the purpose of atom lithography applications. Not only the usual low power regime, where the atoms are focused in the standing light wave, is investigated, but also a higher power regime. In the latter regime the atoms channel through the standing wave, undergoing a damped oscillation around the potential minima of the light field. This is of special interest for lithography, as it allows the production of nanostructures with relative insensitivity to the exact alignment of atomic beam, substrate, and light field. It is, however, also at these higher laser powers that the potential model is expected to break down and the inclusion of the velocity dependence of the force is essential. Experiments in this novel regime with metastable helium atoms have been performed and, in a future paper, the experimental pattern widths will be compared with the calculations presented in this paper. With metastable helium atoms, nanoscale structures can be created in a gold film on a silicon substrate via a two-step process 14]. First, the high internal energy of the focused helium atoms $(20 \mathrm{eV})$ in the $2{ }^{3} \mathrm{~S}_{1}$ metastable state is used to selectively damage an organic resist layer through the standing light wave. Next, the pattern is transferred to the underlying gold film by means of a wet etching process.

In the simulations, the light field is blue detuned from the $2{ }^{3} \mathrm{~S}_{1} \rightarrow 2{ }^{3} \mathrm{P}_{2}$ optical transition $(\lambda=1083 \mathrm{~nm})$ of the helium atom. The atoms are therefore attracted to the intensity minima of the standing light wave, which minimizes spontaneous emission of photons by the atoms. The atomic motion is calculated using only the dipole force. The atomic momentum diffusion due to fluctua- 
tions of the dipole force is neglected. The momentum diffusion is caused by the variation in the number of absorbed and emitted photons, and by the random direction of the spontaneously emitted photons. Furthermore, the atom is assumed to remain in a steady state, i.e., transient effects of the dipole force are neglected. In order to investigate the effects of momentum diffusion and transient effects, some results from Monte Carlo Wave Function (MCWF) calculations similar to Lee 10. are presented as well. These calculations include the velocity dependence of the force, momentum diffusion, atomic diffraction as well as transient effects. However, they are one-dimensional and require significant computational resources.

In the next section, a general expression for the dipole force is derived, and both semi-classical models are outlined. Sec. III describes the method and the parameters of the numerical simulation. The results of the semiclassical simulations are presented in Sec. IV] and they are compared with the MCWF simulations. Finally, concluding remarks are given in Sec. $\mathbf{\nabla}$

\section{THEORETICAL MODELS}

\section{A. Dipole force of a standing wave}

The light force experienced by a two-level atom due to the presence of a classically described light field can be determined by calculating the change in momentum $\vec{p}$ of the atom under influence of the Hamiltonian:

$$
H=H_{A}-\vec{d} \cdot \vec{E}
$$

where $H_{A}$ contains the internal and kinetic energy of the atom and $\vec{d} \cdot \vec{E}$ is the dipole interaction operator that describes the coupling between the atomic dipole moment $\vec{d}$ and the electric field component $\vec{E}$ of the light field. According to the Ehrenfest theorem, the quantum-mechanical analogue of the radiation force is given by the Heisenberg equation of motion

$$
\langle\overrightarrow{\mathcal{F}}\rangle=\frac{d\langle\vec{p}\rangle}{d t}=\frac{i}{\hbar}\langle[H, \vec{p}]\rangle=\langle\nabla(\vec{d} \cdot \vec{E})\rangle=\langle\vec{d}\rangle \cdot \nabla \vec{E} .
$$

In the last step of Eq. (2) the expectation value of the electric field operator $\vec{E}$ is replaced by the value at the atomic centre of mass. This is legitimate in the electric dipole approximation, where the wavelength of the light field $\lambda$ is large compared to the de Broglie wavelength $\lambda_{d B}=\hbar /|\vec{p}|$ of the atom, and spatial variations of the electric field on the scale of the atomic wave-packet can be neglected.

The expectation value of the electric dipole operator $\vec{d}$ can be written in terms of the atomic density matrix $\rho$, which describes the quantum-mechanical state of the two-level atom, as

$$
\begin{aligned}
\langle\vec{d}\rangle & =\operatorname{Tr}(\rho \vec{d})=\vec{d}_{g e}\left(\rho_{g e}+\rho_{e g}\right) \\
& =2 \vec{d}_{g e}(u(t) \cos \omega t-v(t) \sin \omega t),
\end{aligned}
$$

where the atomic density matrix elements $\rho_{g e}$ and $\rho_{e g}=$ $\rho_{g e}^{*}$ are the electronic coherences between the ground state and the excited state of the atom, $\omega$ is the frequency of the radiation field, and $u(t)$ and $v(t)$ are two components of the Bloch vector. In the rotating-wave approximation, where non-resonant terms of the atom-light interactions are neglected, the components of the Bloch vector can be written as

$$
\begin{aligned}
u(t) & =\frac{1}{2}\left(\rho_{g e} e^{i \omega t}+\rho_{e g} e^{-i \omega t}\right), \\
v(t) & =\frac{1}{2 i}\left(\rho_{g e} e^{i \omega t}-\rho_{e g} e^{-i \omega t}\right), \\
w(t) & =\frac{1}{2}\left(\rho_{e e}-\rho_{g g}\right),
\end{aligned}
$$

where the atomic density matrix elements $\rho_{g g}$ and $\rho_{e e}$ are the populations of the ground state and the excited state of the atom, normalized to $\rho_{g g}+\rho_{e e}=1$. For a travelling wave, the electric field component of the light field is given by

$$
\vec{E}(\vec{r}, t)=\vec{\varepsilon}(\vec{r}) E_{0}(\vec{r}) \cos (\omega t+\Phi(\vec{r})),
$$

where $\vec{\varepsilon}(\vec{r}), E_{0}(\vec{r})$, and $\Phi(\vec{r})(=-\vec{k} \cdot \vec{r})$ are the polarization, amplitude and phase of the light wave respectively at the atomic centre-of-mass position $\vec{r}=(x, y, z)$. The Rabi frequency $\Omega(\vec{r})$ of the light field is then defined as

$$
\Omega(\vec{r})=-\frac{\vec{d}_{g e} \cdot \vec{\varepsilon}(\vec{r}) E_{0}(\vec{r})}{\hbar} .
$$

The general expression for the light force (in the electric dipole and rotating-wave approximations) can be written as [15]

$$
\langle\overrightarrow{\mathcal{F}}\rangle=\vec{F}(\vec{r})=-\hbar u_{s t} \nabla \Omega(\vec{r})-\hbar \Omega(\vec{r}) v_{s t} \nabla \Phi(\vec{r}) .
$$

The two parts on the right-hand side of Eq. (7) are the dipole force, proportional to the gradient of the Rabi frequency $\Omega(\vec{r})$, and the scattering force, proportional to the gradient of the phase $\Phi(\vec{r})$ of the light field. The Bloch vector components $u(t)$ and $v(t)$ are replaced by their time-independent steady-state values $u_{s t}$ and $v_{s t}$ respectively. This is valid in the adiabatic approximation, where the atom moves slowly enough in the light field to maintain an equilibrium between its internal state and the radiation field.

The steady-state values can be found by solving the equations of motion of the optical Bloch vector that describe the time evolution of a two-level atom in a light field

$$
\left(\begin{array}{c}
\dot{u} \\
\dot{v} \\
\dot{w}
\end{array}\right)=\left(\begin{array}{ccc}
-\Gamma / 2 & \Delta+\dot{\Phi} & 0 \\
-(\Delta+\dot{\Phi}) & -\Gamma / 2 & -\Omega \\
0 & \Omega & -\Gamma
\end{array}\right)\left(\begin{array}{c}
u \\
v \\
w
\end{array}\right)-\left(\begin{array}{c}
0 \\
0 \\
\Gamma / 2
\end{array}\right),
$$


where $\Gamma / 2 \pi$ is the natural linewidth of the excited state and $\Delta / 2 \pi$ is the detuning between the light field frequency $\omega$ and the frequency of the atomic transition.

The electric field component of a standing-wave light field, composed of an incident and back-reflected Gaussian wave travelling in the $x$-direction, can be written as

$$
\vec{E}(\vec{r}, t)=\vec{\varepsilon} E_{0} \cos (\omega t) \sin (k x) \exp \left(-\frac{y^{2}}{w_{y}^{2}}-\frac{z^{2}}{w_{z}^{2}}\right),
$$

where $k$ is the wave number of the light and $w_{y}$ and $w_{z}$ are the waists of the Gaussian beam profile in the $y$ and $z$ direction respectively. Since this electric field has no phase dependence, the phase gradient term in Eq. (7) vanishes. The general solution of the light force in a standing-wave light field then becomes

$$
\vec{F}_{s w}(\vec{r})=-\hbar u_{s t} \nabla \Omega(\vec{r}) .
$$

The steady-state value of the optical Bloch vector component $u_{s t}$ can be derived using two different approaches; one that neglects the atomic velocity (Sec. IIB), and one that is valid for arbitrary atomic velocities (Sec. IIC).

\section{B. Atom at rest - potential model}

When the transverse velocity of the atom in a standingwave light field is negligible $(\vec{k} \cdot \vec{v} \ll \Gamma)$, the atom travels over a very small distance compared to the optical wavelength $\lambda$ during the relaxation time $\Gamma^{-1}$ of the atom. The optical Bloch equations given in Eq. (8) can then be considered as a set of coupled linear differential equations with time-independent coefficients. The steady-state solutions are found analytically by setting $\dot{u}=\dot{v}=\dot{w}=0$ and they are given by

$$
\begin{aligned}
u_{s t}(\vec{r}) & =\frac{\Delta}{\Omega(\vec{r})} \frac{s(\vec{r})}{1+s(\vec{r})}, \\
v_{s t}(\vec{r}) & =\frac{\Gamma}{2 \Omega(\vec{r})} \frac{s(\vec{r})}{1+s(\vec{r})}, \\
w_{s t}(\vec{r}) & =-\frac{1}{2(1+s(\vec{r}))},
\end{aligned}
$$

where

$$
s(\vec{r})=\frac{2 \Omega^{2}(\vec{r})}{\Gamma^{2}+4 \Delta^{2}}
$$

is the saturation parameter. The final expression for the dipole force acting on an atom at rest in a standing-wave light field, can now be found by combining Eq. (10) and Eq. (111)

$$
\vec{F}_{p o t}(\vec{r})=-\hbar \Delta \frac{\nabla \Omega^{2}(\vec{r})}{2 \Omega^{2}(\vec{r})+\Gamma^{2}+4 \Delta^{2}} .
$$

This force is conservative and it can be written as the gradient of a potential [15, 16]

$$
\vec{F}_{p o t}(\vec{r})=-\nabla U(\vec{r})=-\nabla \frac{\hbar \Delta}{2} \ln [1+s(\vec{r})] .
$$

Eq. (14) is a well-known expression for the dipole force and it is commonly used for semi-classical calculations of atomic motion in a standing-wave light field [1, 2, 9]. Since this force is conservative, the kinetic energy of the atom at any moment is determined by the local potential of the light field.

\section{Moving atom - Minogin model}

When an atom moves with velocity $\vec{v} \neq 0$ in the standing-wave light field, the position of the atom $\vec{r}$ becomes explicitly time dependent as $\vec{r}(t)=\vec{v}$. However, the time dependence of the transverse coordinates $y$ and $z$ can be neglected, since the wavelength of the light $\lambda$ is much smaller than the waist of the Gaussian beam profile. This means that the optical Bloch vector components $u, v$, and $w$ change more rapidly along the axis of the light field ( $x$-direction) than in the transverse directions $y$ and $z$. Therefore, for finding the steady-state solution of the optical Bloch vector components, only the time dependence of the $x$-coordinate has to be taken into account. The Rabi frequency $\Omega(\vec{r})$ can then be written as a periodic function of time

$$
\begin{aligned}
\Omega(\vec{r}, t) & =\Omega_{0}(y, z) \sin (k x) \\
& =\Omega_{0}(y, z) \sin \left(k v_{x} t\right),
\end{aligned}
$$

where $\Omega_{0}(y, z)$ is the peak Rabi frequency at the antinodes of the standing wave, and $v_{x}$ is the velocity of the atom parallel to the axis of the light field. With the Rabi frequency given by Eq. (15), the coefficients of the coupled linear differential equations in Eq. (8) become time dependent. Consequently, the optical Bloch equations can no longer be solved analytically. However, a steadystate solution of $u, v$ and $w$ can be found by expanding each of them in a Fourier series

$$
h(\vec{r})=\sum_{n=-\infty}^{\infty} h_{n}(y, z) e^{i n k x},
$$

where the common notation $h$ is used for $u, v$, and $w$, and the quantities $h_{n}$ satisfy the reality condition $h_{-n}=h_{n}^{*}$. By substituting the Fourier expansions Eq. (16) and the expression for the Rabi frequency given by Eq. (15) in the optical Bloch equations Eq. (8), a set of recursive algebraic equations is obtained for the optical Bloch vector components 11]

$$
\begin{aligned}
\left(\Gamma / 2+i n k v_{x}\right) u_{n} & =\Delta v_{n}, \\
\left(\Gamma / 2+i n k v_{x}\right) v_{n} & =-\Omega_{0}\left(w_{n-1}+w_{n+1}\right)-\Delta u_{n}, \\
\left(\Gamma+i n k v_{x}\right) w_{n} & =\Omega_{0}\left(v_{n-1}+v_{n+1}\right)-\frac{\Gamma}{2} \delta_{n 0} .
\end{aligned}
$$

The dipole force is now also represented in the form of a Fourier series, where the Fourier coefficients can be calculated from the optical Bloch vector components. The expression for the dipole force can be separated into components parallel and perpendicular to the axis of the 
standing-wave light beam ( $x$-direction) as

$$
\begin{aligned}
\vec{F}_{\|}(\vec{r}) & =\vec{F}_{\|}^{0}(\vec{r}) \\
& +\sum_{n=1}^{\infty}(-1)^{n}\left(\vec{F}_{\| u}^{2 n}(\vec{r}) \cos 2 n k x+\vec{F}_{\| v}^{2 n}(\vec{r}) \sin 2 n k x\right)
\end{aligned}
$$

and

$$
\begin{aligned}
\vec{F}_{\perp}(\vec{r}) & =\vec{F}_{\perp}^{0}(\vec{r}) \\
& +\sum_{n=1}^{\infty}(-1)^{n}\left(\vec{F}_{\perp u}^{2 n}(\vec{r}) \cos 2 n k x+\vec{F}_{\perp v}^{2 n}(\vec{r}) \sin 2 n k x\right)
\end{aligned}
$$

where the coefficients of the Fourier series are given by

$$
\begin{aligned}
\vec{F}_{\|}^{0}(\vec{r}) & =-2 \hbar k \Omega_{0} \operatorname{Im} u_{1}, \\
\vec{F}_{\| u}^{2 n}(\vec{r}) & =-2 \hbar k \Omega_{0} \operatorname{Im}\left(u_{2 n+1}-u_{2 n-1}\right), \\
\vec{F}_{\| v}^{2 n}(\vec{r}) & =-2 \hbar k \Omega_{0} \operatorname{Re}\left(u_{2 n+1}-u_{2 n-1}\right),
\end{aligned}
$$

and

$$
\begin{aligned}
\vec{F}_{\perp}^{0}(\vec{r}) & =4 \hbar\left(\frac{y}{w_{y}^{2}}+\frac{z}{w_{z}^{2}}\right) \Omega_{0} \operatorname{Re} u_{1} \\
\vec{F}_{\perp u}^{2 n}(\vec{r}) & =4 \hbar\left(\frac{y}{w_{y}^{2}}+\frac{z}{w_{z}^{2}}\right) \Omega_{0} \operatorname{Re}\left(u_{2 n+1}+u_{2 n-1}\right) \\
\vec{F}_{\perp v}^{2 n}(\vec{r}) & =-4 \hbar\left(\frac{y}{w_{y}^{2}}+\frac{z}{w_{z}^{2}}\right) \Omega_{0} \operatorname{Im}\left(u_{2 n+1}+u_{2 n-1}\right)
\end{aligned}
$$

The coefficients $u_{n}$ are obtained from the solution of the recursion relations (Eq. (17)) in the form of convergent continued fractions. The dipole force given by Eq. (18) and Eq. 19 is dissipative, and the atomic energy is therefore not conserved. The momentum change of the atom can be attributed to an additional damping or heating force, dependent on the intensity of the light field and on the sign of its detuning from atomic resonance.

\section{NUMERICAL SIMULATIONS}

\section{A. Calculation method}

The dipole force derived from the two models described in Sec. IIB and Sec. IC is used to calculate the atomic movement through a standing-wave light field. By straightforward numerical integration of the Newtonian equations of motion, the change of the atomic velocity and position under influence of the dipole force at the current position of the atom in the light field is calculated. In this way a full 3D simulation of the atom trajectories and velocities in the standing-wave light field is performed. The atomic pattern formation is mapped with $2 \mathrm{D}$ atomic distribution plots in the $x y$-plane and in histograms.

The calculation of the coefficients $u_{n}$, required for the calculation of the dipole force in the Minogin model, is described in detail by Minogin and Letokhov [17] and it is summarized for completeness in the appendix. The solutions for the coefficients are found in the form of convergent continued fractions. For the simulations, these continued fractions are calculated with an accuracy better than $10^{-16}$, which requires a maximum of 5000 terms. This precision is necessary to calculate the coefficients $u_{n}$ with an accuracy better than $1 \mathrm{ppm}$. The Fourier series that represent the expression for the dipole force contain at most 2500 Fourier coefficients. A convergence analysis has shown that the dipole force change is negligible at higher accuracies of the coefficients $u_{n}$ and when more terms are added to the Fourier series. Therefore the level of convergence is assumed to be sufficient.

\section{B. Parameters}

The calculations are performed on a beam of helium atoms in the $2{ }^{3} \mathrm{~S}_{1}$ metastable state, which has a lifetime of about $8000 \mathrm{~s}$. The atomic beam has a mean longitudinal velocity of $2000 \mathrm{~m} / \mathrm{s}$ and a longitudinal velocity spread (full width at $1 / e^{2}$ height) of $650 \mathrm{~m} / \mathrm{s}$. After collimation of the beam, the transverse velocity spread of the atoms is reduced to about $3 \mathrm{~m} / \mathrm{s}$. For the calculations, each atom is assigned a longitudinal and transverse initial velocity that is randomly picked from Gaussian velocity distributions with the above described averages and spreads.

The light of the standing wave has a wavelength of $1083 \mathrm{~nm}$, driving the $2{ }^{3} \mathrm{~S}_{1} \rightarrow 2{ }^{3} \mathrm{P}_{2}$ optical transition of the helium atom, which has a natural linewidth $\Gamma / 2 \pi=$ 1.6 MHz. By detuning the light field relatively far to the blue side of the atomic resonance $(\Delta / 2 \pi=375 \mathrm{MHz})$, the atoms are attracted to the nodes of the standingwave light field, which reduces spontaneous emissions. An upper limit for the detuning forms the $2{ }^{3} \mathrm{P}_{1}$ state, which energy level lies $2.3 \mathrm{GHz}$ above the $2{ }^{3} \mathrm{P}_{2}$ state. For very large blue detunings of the light field from the $2{ }^{3} \mathrm{P}_{2}$ state, the atom can thus interact with the light field via the $2{ }^{3} \mathrm{~S}_{1} \rightarrow 2{ }^{3} \mathrm{P}_{1}$ transition.

The Rabi frequency can be calculated from the intensity of the light field as

$$
\Omega(\vec{r})=\Gamma \sqrt{\frac{I(\vec{r})}{2 I_{s a t}}}
$$

where $I_{\text {sat }}=0.17 \mathrm{~mW} / \mathrm{cm}^{2}$ is the saturation intensity of the optical transition and $I(\vec{r})$ is the intensity profile of the standing-wave light field, given by

$$
I(\vec{r})=I_{0} \sin ^{2}(k x) \exp \left(-\frac{2 y^{2}}{w_{y}^{2}}-\frac{2 z^{2}}{w_{z}^{2}}\right) .
$$

The Gaussian light beam has a circular beam profile with a waist $\left(1 / e^{2}\right.$ radius) $w_{y}=w_{z}=331 \mu \mathrm{m}$. The quantity $I_{0}$ is the intensity of the light field at the anti-nodes of 

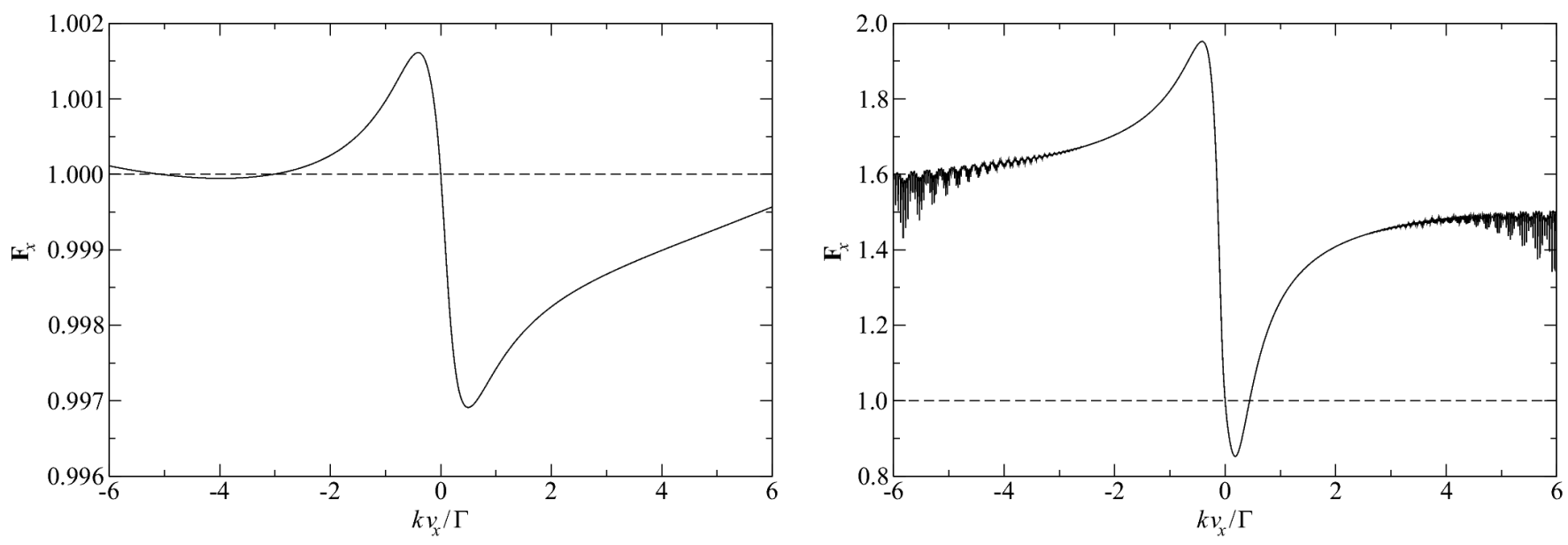

Figure 1: Velocity dependence of the $x$-component of the dipole force of a standing-wave light field in the focusing regime (left) and the channelling regime (right) at position $(x, y, z)=(3 \lambda / 8,0,0)$ in the standing wave and at a detuning of $\Delta / 2 \pi=375 \mathrm{MHz}$. The dipole force according the Minogin model (solid lines) is normalized to the dipole force from the potential model (dashed lines).

the standing wave and it is given by

$$
I_{0}=\frac{8 P_{0}}{\pi w_{y} w_{z}},
$$

where $P_{0}$ is the power of the incident light beam. Depending on this power, two different regimes can be distinguished for guiding the atoms through the standingwave light field. At low power, the atoms can be focused at the centre of the Gaussian light beam. For large detunings $(\Delta \gg \Gamma)$, the power required for this focusing can be calculated from 9 ]

$$
P_{0}=5.37 \frac{\pi m \vec{v}^{2} \Delta I_{s a t}}{2 \hbar \Gamma^{2} k^{2}} .
$$

This focusing power is independent of the waist of the light beam. For the conditions mentioned above, $P_{0}=$ $2.4 \mathrm{~mW}$. For high-power light fields, the atoms oscillate through a potential minimum of the standing wave and the sign of the transverse velocity of the atoms changes many times. This is called the channelling regime. For calculations in this regime a power $P_{0}=800 \mathrm{~mW}$ is used. The dipole force in the channelling regime is then about one order of magnitude larger than in the focusing regime.

\section{The dipole force}

The velocity dependence of the $x$-component of the dipole force in the focusing and channelling regime for both models is depicted in Fig. 1. The graphs show that, in the focusing regime (left graph), the difference between the dipole force according to the potential model and the Minogin model is very small (at most $0.3 \%$ ). In the channelling regime (right graph), however, the dipole force of the two models differs significantly. The force shows a negative slope for small transverse velocities $\left(\left|k v_{x} / \Gamma\right|<0.2\right)$, which means that the force is a damping force. This cooling effect is essentially the "blue-detuned Sisyphus cooling" introduced by Dalibard and CohenTannoudji [18]. At larger velocities, the dipole force in the Minogin model is significantly larger than in the potential model. It is therefore expected that the distinction between the two models will be most pronounced in the channelling regime. Furthermore, in the channelling regime, the Minogin model shows some resonance peaks at high atom velocities. These are called Doppleron resonances [19], and occur when the atom is excited to the $2{ }^{3} \mathrm{P}_{2}$ state by multiple photon absorptions and emissions. When an atom absorbs $(n+1)$ photons from one wave of the standing-wave light field, and emits $n$ photons into the other, these resonances appear at velocities

$$
k v_{x} / \Gamma= \pm \frac{\Delta}{(2 n+1) \Gamma}
$$

\section{RESULTS}

Fig. 2] shows a projection on the $x z$-plane of the atomic motion through the standing-wave light field in the focusing regime (upper graphs) and channelling regime (lower graphs). The centre of the Gaussian light beam, which propagates in the $x$-direction, is located at $z=0$. The graphs cover half a wavelength $(\lambda / 2=542 \mathrm{~nm})$ of the standing-wave light field, showing exactly one potential minimum through which the atoms travel in the positive $z$-direction (from left to right). To allow the atoms to experience a maximum dipole force, the starting $y$-position is $y=0$ for all atoms. However, since the simulations are $3 \mathrm{D}$ and the atoms have an initial velocity in the $y$ - 

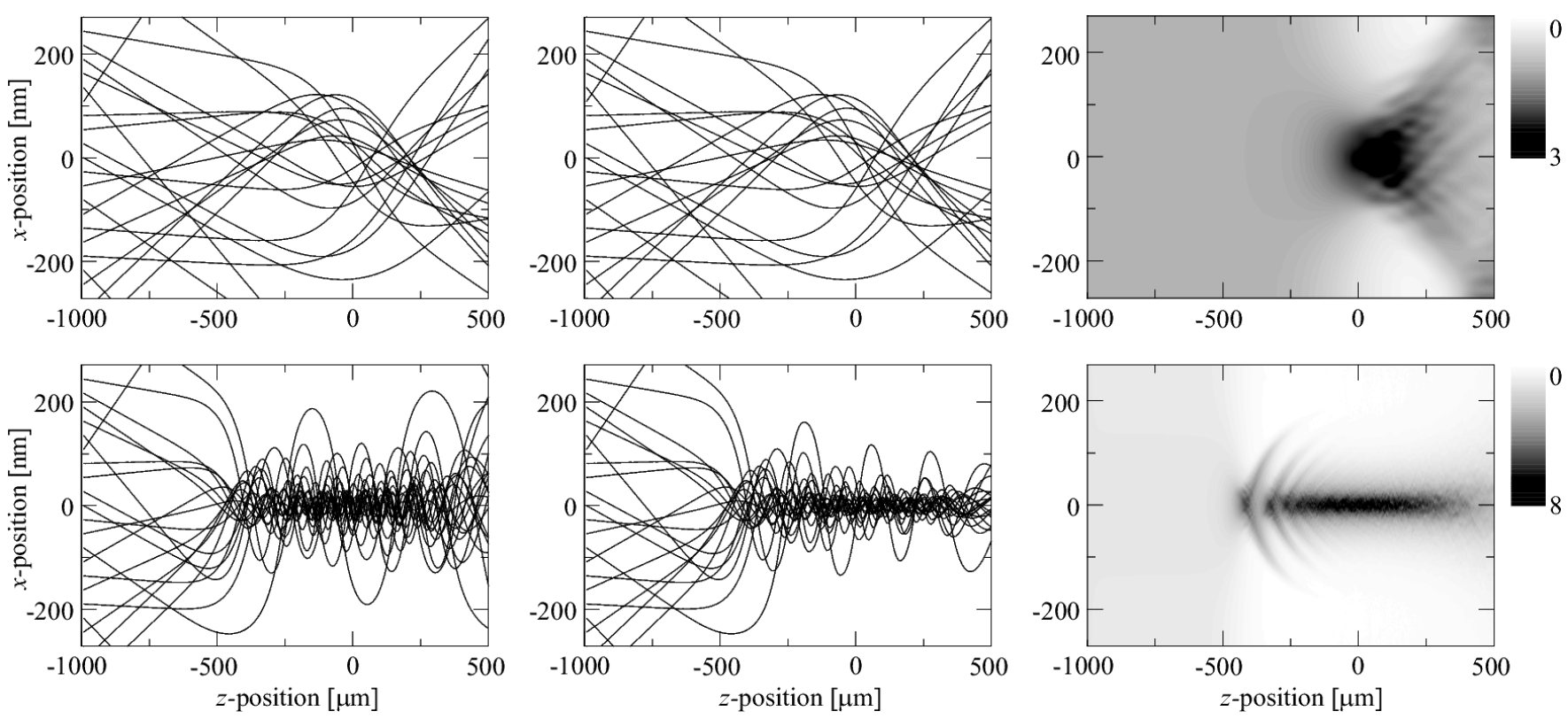

Figure 2: Atom trajectories through the standing-wave light field in the focusing regime (upper graphs) and the channelling regime (lower graphs). Semi-classical calculations are performed with the potential model (left) and the Minogin model (centre). The right graphs show atomic density distributions of Monte Carlo Wave Function (MCWF) simulations.

direction, this position is not maintained while travelling through the light field.

Since the velocity dependence of the dipole force in the focusing regime is almost negligible (see Fig. 1), the atomic motion in this regime according to the potential model (upper left graph) is very similar to the calculation with the Minogin model (upper centre graph). In the channelling regime, the calculated trajectories differ considerably for the potential model (lower left graph) and the Minogin model (lower centre graph), due to the higher average value and the strong velocity dependence of the dipole force in the latter model (see Fig. 1). The potential model is invalid in this regime, since the atoms have a transverse velocity spread of $3 \mathrm{~m} / \mathrm{s}\left(k v_{x} / \Gamma=1.7\right)$, and the transverse velocity of the atoms can even become on the order of $10 \mathrm{~m} / \mathrm{s}$ in the standing wave. In the channelling graph of Fig. 2 the additional damping force results in an increased confinement of the atoms in the potential well of the standing wave. The upper right and lower right graphs of Fig. 2] show one-dimensional MCWF calculations for the focusing regime and channelling regime respectively. In these calculations atomic diffraction, velocity dependence and momentum diffusion are fully incorporated. The plots show the atomic density distribution on a grey-scale for 225 quantum trajectories, corresponding to the same initial distribution as used for the semi-classical simulations. The characteristic feather-like structures in the channelling regime (lower right graph) do not constitute a specific quantum feature. They correspond to (approximate) caustics in the semi-classical trajectories and they become clearly visible if a larger number of trajectories is plotted in the
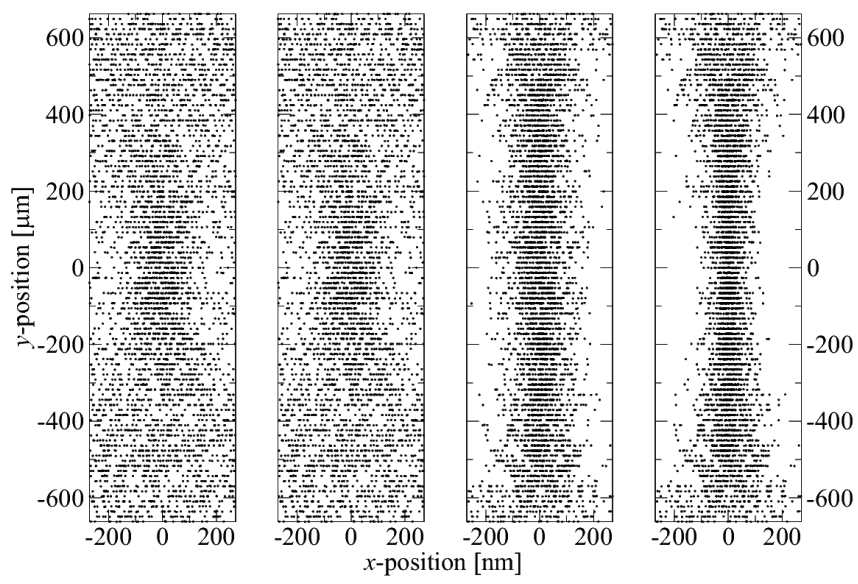

Figure 3: Distribution plots of the positions where the atoms hit the sample after travelling through the standing-wave light field at sample position $z=w_{z} / 4=83 \mu \mathrm{m}$. From left to right, the first to graphs show plots of the focusing regime using the potential model (first graph) and the Minogin model (second graph). The last two graphs show the channelling regime using the potential model (third graph) and the Minogin model (fourth graph).

same way as in the lower centre graph.

Distribution plots of the atoms are shown in Fig. 3] Every dot in these graphs represents a position where the atom has hit the sample after travelling through the standing-wave light field. The calculations are performed on atoms in a lattice of $101 \times 101$ atoms. The starting positions of the atoms range from $(x, y)=\left(-\lambda / 2,-2 w_{y}\right)$ to $(x, y)=\left(\lambda / 2,2 w_{y}\right)$. The plots only show half of this 

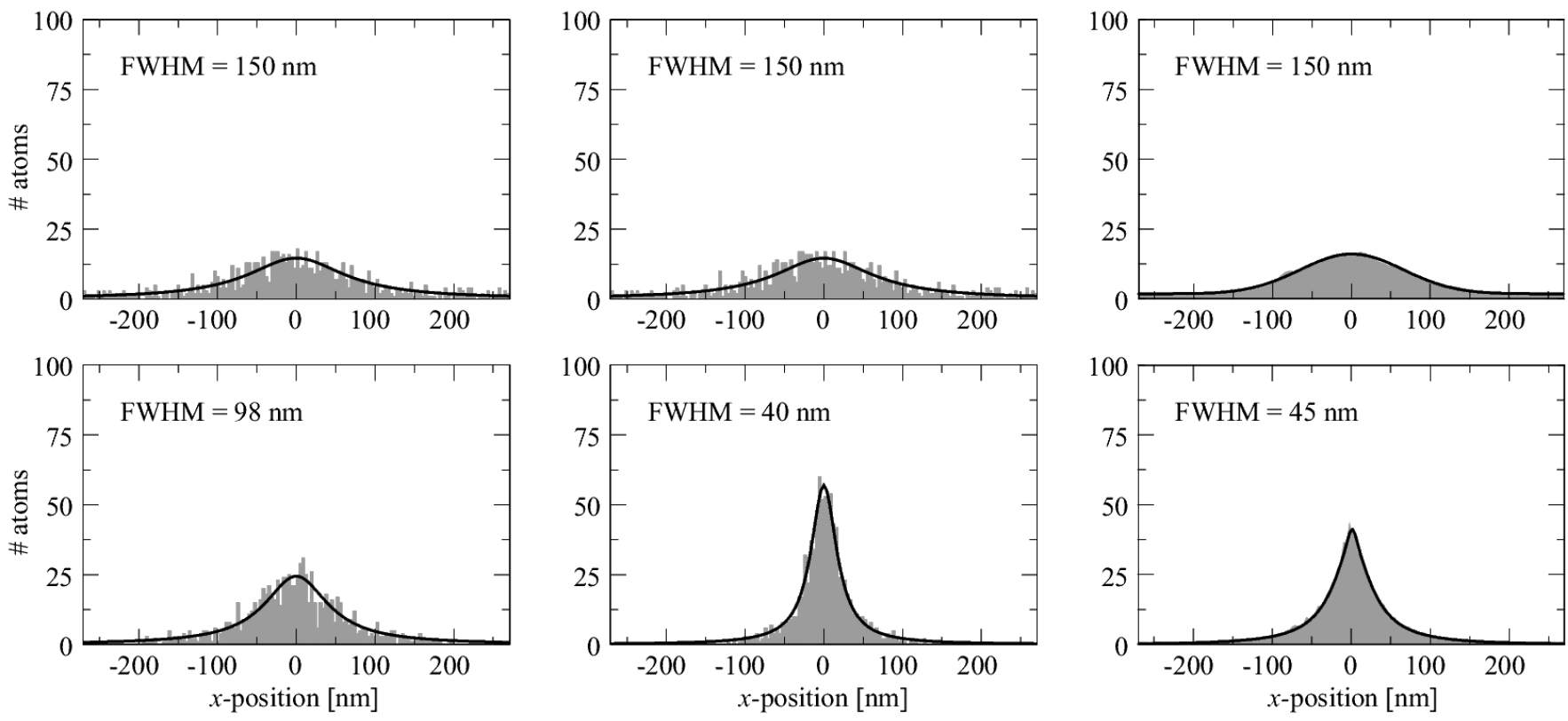

Figure 4: Histograms of the atom distributions taken at $z=w_{z} / 4=83 \mu \mathrm{m}$. The upper graphs show the distributions for the focusing regime in the potential model (left), Minogin model (centre), and the MCWF simulations (right). Similarly, the channelling regime is represented by the lower graphs.

window in the $x$-direction, from $x=-\lambda / 4$ to $x=\lambda / 4$. In the focusing regime, the light-field does not act as a perfect lens, but shows some abberations. This imperfection is enhanced by the longitudinal and transverse velocity spread of the atoms. As a result, the best position to place the sample is not at the centre of the light beam $(z=0)$, but slightly behind it. For the channelling regime, the sample position is far less critical, but the best results are at similar positions. Therefore, the comparison of the two regimes for the different models in Fig. [3 is performed at $z=w_{z} / 4=83 \mu \mathrm{m}$, where optimal results are expected.

The first two plots show the atom distributions in the focusing regime for the potential model (first plot) and the Minogin model (second plot). As expected from the comparison of the trajectories in this regime, shown in the upper graphs of Fig. 2 the differences between the two models are negligible. The distributions in the channelling regime with the potential model (third plot) and the Minogin model (fourth plot) show that the atoms are confined for a wider range along the $y$-axis as compared to the focusing regime. Furthermore, for the Minogin model, the atoms are more localized to the nodes of the standing wave.

To make a more quantitative comparison of the four plots of Fig. 3 histograms of the atom distributions are presented in Fig. 4 Only the atoms located between $y=-100 \mu \mathrm{m}$ and $y=100 \mu \mathrm{m}$ are taken into account for the histograms, because atoms outside this region do not contribute to the desired pattern (in the focusing regime). The black curves through the histograms are Lorentzian fits from which the Full Width at Half
Maximum (FWHM) of the distribution can be deduced. The upper graphs, that represent the calculations in the focusing regime, show that the FWHM of the distribution is $150 \mathrm{~nm}$ for both semi-classical models as well as for the quantum-mechanical model. For the calculations in the channelling regime (lower graphs), the distribution of the potential model (left graph) has a FWHM of $98 \mathrm{~nm}$. This width deviates clearly from the distribution of the Minogin model (centre graph), which has a FWHM of $40 \mathrm{~nm}$. The smaller width of this distribution can be explained from the additional cooling force that is included in the Minogin model. The distributions for the full quantum-mechanical calculations are shown in the right graphs. The FWHM of the central peak is $45 \mathrm{~nm}$, somewhat larger than the results from the continued fraction semi-classical results. This larger width is mostly due to the fact that the steady-state situation for the atoms is not completely realized during the interaction time. Achieving a steady state requires a number of spontaneous emissions per atom. Due to the large detuning of the light field from atomic resonance, and due to the fact that the atoms are channelled in the region of low light intensity, almost one half of the atoms never undergoes a spontaneous emission. The influence of diffusion is seen in the wings of the MCWF distributions. However, overall the MCWF results agree very well with the semi-classical calculations using the Minogin model, confirming the large reduction in the FWHM of the distribution due to the cooling force. 


\section{CONCLUSIONS}

The simulations have shown that nanoscale patterns can be created utilizing the optical dipole force to guide atoms through a standing-wave light field. For the calculation of the atomic motion, two models have been applied: a simple one that uses the conventional dipole force, derived from a potential, and another one that includes the velocity dependence of this dipole force. In the 'conventional' focusing regime, where a low-power light field is used, the differences between the potential model and the Minogin model are negligible. Both models show that in principle nanoscale pattern sizes of $150 \mathrm{~nm}$ can be achieved with the specified experimental parameters. It should be noted, that these parameters are not optimized for focusing: using a smaller laser focus and more laser power, much tighter focusing can be achieved without entering the channelling regime. However, the sample position is very critical, and good focusing is only achieved for atoms that pass the light field close to its centre. The high-power channelling regime, that we are presently using for nanostructure production with metastable helium atoms, is more robust. It is therefore better suitable for creating narrow patterns. In this regime, the Minogin model shows strong deviations from the potential model, due to the contribution of velocity dependent terms to the dipole force. Because of the large transverse velocity $\left(k v_{x}>\Gamma\right)$ of the atoms in the standing-wave light field, the potential model breaks down and should not be applied for calculations in this regime. The results of the calculations with the Minogin model show structures with a FWHM of $40 \mathrm{~nm}$. This model does not take into account the momentum diffusion and transient effects, which leads to broadening of the structure size, as is shown by the MCWF simulations. However, the MCWF simulations support the results of the Minogin model by showing a better confinement of the atoms in the standing-wave light field than expected from calculations with the conventional model. This is due to an additional cooling force that is not incorporated in the conventional model.

\section{Acknowledgements}

Financial support from the Foundation for Fundamental Research on Matter (FOM) is gratefully acknowledged.
[1] G. Timp, R.E. Behringer, D.M. Tennant, J.E. Cunningham, M. Prentiss, K.K. Berggren, Phys. Rev. Lett. 69, 1636 (1992)

[2] J.J. McClelland, R.E. Scholten, E.C. Palm, R.J. Celotta, Science 262, 877 (1993)

[3] R.W. McGowan, D.M. Giltner, S.A. Lee, Opt. Lett. 20, 2535 (1995)

[4] F. Lison, H.J. Adams, D. Haubrich, M. Kreis, S. Nowak, D. Meschede, Appl. Phys. B 65, 419 (1997)

[5] K.S. Johnson, J.H. Thywissen, N.H. Dekker, K.K. Berggren, A.P. Chu, R. Younkin, M. Prentiss, Science 280, 1583 (1998)

[6] P. Engels, S. Salewski, H. Levsen, K. Sengstock, W. Ertmer, Appl. Phys. B 69, 407 (1999)

[7] D. Meschede, H. Metcalf, J. Phys. D 36, R17 (2003)

[8] K.K. Berggren, M. Prentiss, G.L. Timp, R.E. Behringer, J. Opt. Soc. Am. B 11, 1166 (1994)

[9] J.J. McClelland, J. Opt. Soc. Am. B 12, 1761 (1995)

[10] C.J. Lee, Phys. Rev. A 61, 063604 (2000)

[11] V.G. Minogin, O.T. Serimaa, Opt. Commun. 30, 373 (1979)

[12] Q. Li, B.W. Stenlake, I.C.M. Littler, H.-A. Bachor, K.G.H. Baldwin, D.E. McClelland, Laser Phys. 4, 983 (1994)

[13] Q. Li, K.G.H. Baldwin, H.-A. Bachor, D.E. McClelland, J. Opt. Soc. Am. B 13, 257 (1996)

[14] S. Nowak, T. Pfau, J. Mlynek, Appl. Phys. B 63, 203 (1996)

[15] R.J. Cook, Phys. Rev. A 20, 224 (1979)

[16] A. Ashkin, Phys. Rev. Lett. 40, 729 (1978)

[17] V.G. Minogin, V.S. Letokhov, Laser light pressure on atoms (Gordon and Breach, New York, 1987)

[18] J. Dalibard, C. Cohen-Tannoudji, J. Opt. Soc. Am. B 2,
$1707(1985)$

[19] E. Kyrölä, S. Stenholm, Opt. Commun. 22, 123 (1977)

\section{Appendix A: CONTINUED FRACTION METHOD}

The determination of the dipole force in the Minogin model, given by Eqs. (18) and (19), requires the optical Bloch vector components $u_{n}$, that are embedded in the expressions of the Fourier coefficients in Eqs. (20) and (21). The coefficients $u_{n}$ can be calculated with a continued fraction method according to Minogin and Letokhov [17]. A summary of this method is described below.

The upper expression of Eq. (17) can be rewritten in the form

$$
u_{n}=\frac{\Delta}{\Gamma / 2+i n k v_{x}} v_{n}
$$

Since $v_{n}$ is non-zero for odd $n$, and $w_{n}$ is non-zero for even $n$, the middle and lower expressions of Eq. (17) can be combined to a single expression as

$$
b_{n}-D_{n}\left(b_{n-1}+b_{n+1}\right)=-\delta_{n 0} / 2,
$$

where

$$
b_{n}= \begin{cases}v_{n} & \text { for odd } n, \\ w_{n} & \text { for even } n,\end{cases}
$$


and the coefficients $D_{n}$ are given by

$$
D_{n}= \begin{cases}-\frac{\Omega\left(\Gamma / 2+i n k v_{x}\right)}{\left(\Gamma / 2+i n k v_{x}\right)^{2}+\Delta^{2}} & \text { for odd } n, \\ \frac{\Omega}{\Gamma+i n k v_{x}} & \text { for even } n .\end{cases}
$$

The coefficients $b_{n}$ fulfil the reality condition

$$
b_{-n}=b_{n}^{*},
$$

and therefore only non-negative integers $(n \geq 0)$ have to be considered. The relation between two successive quantities $b_{n}$ and $b_{n+1}$ can then be found by substituting

$$
b_{n+1}=q_{n} b_{n}
$$

into Eq. A22). This leads to a recursion relation for $q_{n}$ given by

$$
q_{n}=\frac{1}{D_{n}}-\frac{1}{q_{n-1}} .
$$

The quantity $q_{0}$ can now be expressed as a convergent continued fraction

$$
q_{0}=\frac{D_{1}}{1+\frac{p_{1}}{1+\frac{p_{2}}{1+\frac{p_{3}}{1+\ldots}}}}
$$

where

$$
p_{n}=-D_{n} D_{n+1} .
$$

The quantity $b_{0}=w_{0}$ is found by solving Eq. A2 for $n=0$ and gives

$$
b_{0}=\frac{1}{4 \Omega / \Gamma \operatorname{Re}\left(q_{0}\right)-2} .
$$

From Eq. A6 and this initial coefficient $b_{0}$, all successive coefficients $b_{n}$ can be calculated. By substituting the coefficients $b_{n}\left(=v_{n}\right.$ for odd $\left.n\right)$ into Eq. A1), the coefficients $u_{n}$ can be found straightforwardly. 